\title{
Article \\ Geometry of $k$-Yamabe Solitons on Euclidean Spaces and Its Applications to Concurrent Vector Fields
}

\author{
Akram Ali $^{1}$ (D), Fatemah Mofarreh $^{2}$ (D) Pişcoran Laurian-Ioan ${ }^{3, *}$ (D) and Nadia Alluhaibi ${ }^{4}$ (D) \\ 1 Department of Mathematics, College of Science, King Khalid University, Abha 62529, Saudi Arabia; \\ akali@kku.edu.sa \\ 2 Mathematical Science Department, Faculty of Science, Princess Nourah bint Abdulrahman University, \\ Riyadh 11546, Saudi Arabia; fyalmofarrah@pnu.edu.sa \\ 3 Department of Mathematics and Computer Science Victoriei 76, North University Center of Baia Mare \\ Technical University of Cluj Napoca, 430122 Baia Mare, Romania \\ 4 Department of Mathematics, Science and Arts College, Rabigh Campus, King Abdulaziz University, \\ Jeddah 21911, Saudi Arabia; nallehaibi@kau.edu.sa \\ * Correspondence: plaurian@yahoo.com
}

check for

updates

Citation: Ali, A.; Mofarreh, F.;

Alluhaibi, N.; Laurian-Ioan, P.

Geometry of $k$-Yamabe Solitons on

Euclidean Spaces and Its

Applications to Concurrent Vector

Fields. Symmetry 2021, 13, 222.

https://doi.org/10.3390/sym13020222

Academic Editor: Palle E. T. Jorgensen

Received: 31 December 2020

Accepted: 26 January 2021

Published: 29 January 2021

Publisher's Note: MDPI stays neutral with regard to jurisdictional claims in published maps and institutional affiliations.

Copyright: () 2021 by the authors. Licensee MDPI, Basel, Switzerland. This article is an open access article distributed under the terms and conditions of the Creative Commons Attribution (CC BY) license (https: / / creativecommons.org/ licenses/by/4.0/).
Abstract: In this paper, we give some classifications of the $k$-Yamabe solitons on the hypersurfaces of the Euclidean spaces from the vector field point of view. In several results on $k$-Yamabe solitons with a concurrent vector field on submanifolds in Riemannian manifolds, is proved that a $k$-Yamabe soliton $\left(M^{n}, g, v^{T}, \lambda\right)$ on a hypersurface in the Euclidean space $\mathbb{R}^{n+1}$ is contained either in a hypersphere or a hyperplane. We provide an example to support this study and all of the results in this paper can be implemented to Yamabe solitons for $k$-curvature with $k=1$.

Keywords: concurrent vector fields; $k$-Yamabe solitons; hypersurface; Euclidean spaces

\section{Introduction and Motivations}

The geometric flows is an interesting tool in geometric analysis from the singularities point of view. In this respect, we can study an extension of the scalar curvature $R$ which is a $k$-curvature. If $k=1$, then $k$-curvature is a usual scalar curvature $R$. This concept is defined in [1] between the Schouten tensor $A_{g}=\frac{1}{n-2}\left(R i c-\frac{R}{2(n-1)} g\right)$ and the Weyl tensor $W$, exists the following relation: $R m=A_{g} \odot g+W$. Here the symbol $\odot$ denotes the KulkarniNomizu product Therefore, the metric $g$ associated with $k$-curvature is connected as

$$
\sigma_{k}(g)=\sigma_{k}\left(\mu\left(g^{-1} A_{g}\right)\right)=\sum_{i_{1}<\cdots<i_{k}} \mu_{i_{1}} \cdots \mu_{i_{k}}, \quad \text { for } 1 \leq k \leq n,
$$

where $\mu\left(g^{-1} A_{g}\right)=\left(\mu_{1} \cdots \mu_{n}\right)$ is a collection eigenvalue of $g^{-1} A_{g}$ and $\sigma_{k}(\mu)=\sum_{i_{1}<\cdots<i_{k}}$ $\mu_{i_{1}} \cdots \mu_{i_{k}}$. In this case, for a locally conformally flat manifold $\left(M^{n}, g\right)$, we take into account the geometric flow

$$
\frac{d}{d t} g(t)=-\left(\log \sigma_{k}(g(t))-\log r_{k}(g(t))\right) g(t) \quad \text { and } \quad g(0)=g_{0}
$$

such that $r_{k}(g(t))=\exp \left(\frac{1}{\operatorname{vol}(g(t))} \int \log \sigma_{k}(g(t)) d v o l_{g(t)}\right)$ and $\sigma_{k}(g(t))$ is positive. If the flow (1) has a self-similar solution, then it is called $k$-Yamabe soliton and it is defined as follows:

$$
\mathcal{L}_{X} g=2\left(\log \sigma_{k}(g)-\lambda\right) g
$$


where $\lambda$ is a constant and $X$ is a vector field. The $k$-Yamabe solitons can be classified as expanding, steady or shrinking according to the following values of $\lambda: \lambda<0, \lambda=$ 0 , or $\lambda>0$. If $X$ is a gradient vector field $X=\nabla f$, then (2) is equivalent to the following

$$
\nabla^{2} f=\left(\log \sigma_{k}(g)-\lambda\right) g
$$

In this case, $\left(M^{n}, g\right)$ is recognized as a gradient of the $k$-Yamabe soliton. For more details, please see $[2,3]$. It is exciting to know that the concurrent vector field plays a crucial role in classified Riemannian geometry. For example, Chen-Deshmukh in [4] gave a classification of the Ricci soliton associated with the concurrent vector field and also, they proved many new and important results. They proved that every Ricci soliton $\left(M^{n}, g, v, \lambda\right)$ associated with a concurrent potential vector field $v$ is a gradient and also that any Ricci soliton $\left(M^{n}, g, v^{T}, \lambda\right)$ on a totally umbilical submanifold is a trivial Ricci soliton. From the viewpoint of the vector fields on a hypersurface, Chen-Deshmukh [5] have classified the Ricci solitons on some Euclidean hypersurfaces. In the same pattern, Yamabe soliton is considered a very influential soliton type. Therefore, $k$-Yamabe soliton has also become useful in the geometric analysis because it is a generalization of Yamabe soliton for $k=1$. For instance, in [2], the authors acknowledged the full nonlinear Yamabe flow. They proved that on a closed locally conformally flat manifold, if the $k$-curvature is positive for the $k$-Yamabe soliton, it must have constant $\sigma_{k}$-curvature. It was shown that if the first nonzero eigenvalue $\mu_{1}$ satisfied $\mu_{1} \leq \frac{R}{n-1}$ for a compact $k$-Yamabe soliton having constant scalar curvature, then $k$-Yamabe soliton is trivial [2]. More triviality results can be found in [3], that is, every compact gradient $k$-Yamabe soliton must have constant $k$-curvature and certain conditions over the gradient. On the other hand, Yamabe solitons and quasi-Yamabe solitons with concurrent vector fields are discussed in [6] and also in a great number of good results in [7-18]. Motivated by some previous results regarding the classification of the theory of solitons geometry; we shall study some geometric classifications notes for $k$-Yamabe solitons on Euclidean hypersurfaces, if it is a potential field, originated from their position vector fields.

\section{Background and Notations}

Assuming $M^{n}$ is isometrically immersed into a Riemannian manifold $\widetilde{M}^{m}$ among induced connections $\nabla^{\perp}$ and $\nabla$ on the normal bundle $T^{\perp} M$ and the tangent bundle $T M$ of $M^{n}$, in the same order, then the Weingarten and Gauss formulae are

$$
\begin{array}{r}
\widetilde{\nabla}_{W_{1}} \xi=-A_{\xi} W_{1}+\nabla_{W_{1}}^{\perp} \xi \\
\widetilde{\nabla}_{W_{1}} W_{2}=\nabla_{W_{1}} W_{2}+h\left(W_{1}, W_{2}\right)
\end{array}
$$

for each $W_{1}, W_{2} \in \mathfrak{X}(T M)$ and $\xi \in \mathfrak{X}\left(T^{\perp} M\right)$. Furthermore, $A_{\xi}$ and $h$ are the shape operator and respectively the second fundamental form for an embedding of $M^{n}$ into $\widetilde{M}^{m}$. The relation between both of them can be given as follows:

$$
g\left(h\left(W_{1}, W_{2}\right), \xi\right)=g\left(A_{\xi} W_{1}, W_{2}\right) .
$$

The Gauss equation for a submanifold $M^{n}$ is given by:

$$
\widetilde{R}\left(W_{1}, W_{2}, Y_{1}, Y_{2}\right)=R\left(W_{1}, W_{2}, Y_{1}, Y_{2}\right)+g\left(h\left(W_{1}, Y_{1}\right), h\left(W_{2}, Y_{2}\right)\right)-g\left(h\left(W_{1}, Y_{2}\right), h\left(W_{2}, Y_{1}\right)\right)
$$

for any $W_{1}, W_{2}, Y_{1}, Y_{2} \in \mathfrak{X}(T M)$, where the curvature tensors on $\tilde{M}^{m}$ and $M^{n}$ are denoted by $\widetilde{R}$ and $R$, respectively. Now we give some classifications of submanifold $M^{n}$ as follows:

(i) The mean curvature $\mathbf{H}$ of $M^{n}$ in $\widetilde{M}^{m}$ is expressed as $\mathbf{H}=\frac{1}{n} \operatorname{trace}(h)$. If $\mathbf{H}=0$, then $M^{n}$ is minimal in $\tilde{M}^{m}$ [19].

(ii) If for $h$, holds the following relation $h\left(X_{1}, X_{2}\right)=g\left(X_{1}, X_{2}\right) \mathbf{H}$, then $M^{n}$ is totally umbilical. It is referred to be totally geodesic when $h=0$. 
(iii) Let the shape operator be endowed with the eigenvalue of multiplicity $\delta$. If this condition holds, a hypersurface of $(n+1)$-dimensional $\mathbb{R}^{n+1}$ is said to be a quasiumbilical hypersurface. On the subset $\mu$ of $M^{n}$ such that $\operatorname{mult}(\delta)=n-1$, a characterized direction of an quasiumbilical hypersurface has an eigenvector with the eigenvalue of multiplicity one.

(iv) Let $M$ be a smooth $n$-dimensional manifold. A smooth map $\psi: M \longrightarrow \mathbb{R}^{n+1}$ is a hypersurface (an immersion) if its differential is injective. It is an embedding if it is also a homeomorphism onto its image $\psi(M)$. In this case, it is called orientation hypersuface of $M$ [20].

The gradient and the Hessian positive function $\psi$ defined on $M^{n}$ can be written as follows:

$$
\begin{gathered}
\nabla \psi=\sum_{i=1}^{n} e_{i}(\psi) e_{i}, \quad \text { and }\|\nabla \psi\|^{2}=\sum_{i=1}^{n}\left((\psi) e_{i}\right)^{2} . \\
H^{\psi}\left(e_{i}, e_{j}\right)=e_{i}\left(e_{j} \psi\right)-\left(\nabla_{e_{i}} e_{j}\right) \psi
\end{gathered}
$$

If $\Psi:(M, g) \longrightarrow \mathbb{R}^{m}$ is an isometric embedding from the Riemannian submanifold $M^{n}$ with $\operatorname{dim} M=n$ into an Euclidean space $\mathbb{R}^{m}$ with $\operatorname{dim} \mathbb{R}^{m}=m$, then the components of a position vector $v$ of $M^{n}$ in $\mathbb{R}^{m}$ are decomposed as follows:

$$
v=v^{T}+v^{\xi}
$$

where $v^{T}$ and $v^{\xi}$ are tangential and respectively, the normal components of $v$. Another interesting theorem that called the Hodge-de Rham decomposition theorem [21]. It is stated

Theorem 1 ([21]). Any vector field X a compact oriented Riemannian manifold $M$ can be decomposed as the sum of a divergence free vector field $Y$ and the gradient of a function $\omega$ such that

$$
X=\nabla \omega+Y
$$

where div $Y=0$. and $\omega$ is the Hodge-de Rham potential function.

The relation between Lie derivatives of $X$ and $Y$ is defined as

$$
\frac{1}{2} \mathcal{L}_{X g}=\nabla^{2} \omega+\frac{1}{2} \mathcal{L}_{Y} g
$$

\section{Main Results}

Now we are going to give our new results.

Theorem 2. A submanifold $\left(M^{n}, g\right)$ of an Euclidean space $\mathbb{R}^{m}$ is a $k$-Yamabe soliton endowed with $v^{T}$ as its soliton vector, if and only if $h$ satisfies:

$$
\tilde{g}\left(h\left(W_{1}, W_{2}\right), v^{\xi}\right)=\left(\log \sigma_{k}(g)-\lambda-1\right) g\left(W_{1}, W_{2}\right)
$$

for any $W_{1}, W_{2} \in \mathfrak{X}(M)$.

Proof. The position vector $v$ of the manifold $M^{n}$ from $\mathbb{R}^{m}$ is a concurrent vector field, therefore it satisfies

$$
\tilde{\nabla}_{W_{1}} v=W_{1}
$$

Using (9) in the above equation, we have

$$
\tilde{\nabla}_{W_{1}} v^{T}+\tilde{\nabla}_{W_{1}} v^{\xi}=W_{1}
$$


Now making use of the Weingarten (4) and the Gauss (5) formulas in the above equation, one obtains:

$$
\nabla_{W_{1}} v^{T}+h\left(W_{1}, v^{T}\right)-A_{v^{\xi}} W_{1}+\nabla_{W_{1}}^{\perp} v^{\tau}=W_{1} .
$$

Equating the normal and tangential components in the previous equations, we find that:

$$
h\left(W_{1}, v^{T}\right)=-\nabla_{W_{1}}^{\perp} v^{\xi} \text { and } \nabla_{W_{1}} v^{T}=A_{v^{\xi}} W_{1}+W_{1} .
$$

On the other hand, applying Lie derivative definition, one obtains:

$$
\left(\mathcal{L}_{v^{T}} g\right)\left(W_{1}, W_{2}\right)=g\left(\nabla_{W_{1}} v^{T}, W_{2}\right)+g\left(\nabla_{W_{2}} v^{T}, W_{1}\right) .
$$

From (2) and (14), it implies that

$$
\begin{aligned}
2\left(\log \sigma_{k}(g)-\lambda\right) g\left(W_{1}, W_{2}\right) & =g\left(W_{1}, W_{2}\right)+g\left(A_{v \xi} W_{1}, W_{2}\right) \\
& +g\left(A_{v \xi} W_{2}, W_{1}\right)+g\left(W_{1}, W_{2}\right) .
\end{aligned}
$$

The above equation is equivalent with:

$$
2\left(\log \sigma_{k}(g)-\lambda\right) g\left(W_{1}, W_{2}\right)=2 \tilde{g}\left(W_{1}, W_{2}\right)+2 \tilde{g}\left(h\left(W_{1}, W_{2}\right), v^{\tilde{\xi}}\right)
$$

for any $W_{1}, W_{2}$ which are tangent to $M^{n}$. From Equations (2) and (17), we find that $M^{n}$ is a $k$-Yamabe soliton with soliton vector $v^{T}$ if and only if condition (12) is satisfied.

The following result is a consequences of Theorem 2.

Corollary 1. An isometric embedding $\Psi: M^{n} \longrightarrow \mathbb{S}^{m-1} \subset \mathbb{R}^{m}$ of $M^{n}$ into the hypersurface $\mathbb{S}^{m-1}$ with center o and radius $r$, is a $k$-Yamabe soliton. Here, $v^{T}$ is a soliton vector field if and only if $\sigma_{k}$-curvature $\log \sigma_{k}(g)$ of $\left(M^{n}, g\right)$ is constant.

Proof. For an isometric embedding $\Psi: M^{n} \longrightarrow \mathbb{S}^{m-1} \subset \mathbb{R}^{m}$ of $M^{n}$ into the $\mathbb{S}^{m-1}$, we have $v^{\tau}=v$. Here, the second fundamental form $h$ of $M^{n}$ in $\mathbb{S}^{m-1}$ holds

$$
h\left(W_{1}, W_{2}\right)=h^{\prime}\left(W_{1}, W_{2}\right)-\frac{g\left(W_{1}, W_{2}\right)}{r^{2}} v
$$

which follows from the Lemma 3.5 in [22]. Taking the inner product with $v^{\xi}$ in (18), one obtains

$$
\tilde{g}\left(h\left(W_{1}, W_{2}\right), v^{\tilde{\xi}}\right)=\tilde{g}\left(h^{\prime}\left(W_{1}, W_{2}\right), v^{\tilde{\xi}}\right)-\frac{g\left(W_{1}, W_{2}\right)}{r^{2}} g\left(v, v^{\tau}\right)
$$

which implies that because $v=v^{\xi}$, one obtains:

$$
\tilde{g}\left(h\left(W_{1}, W_{2}\right), v^{\tilde{\xi}}\right)=-g\left(W_{1}, W_{2}\right) .
$$

Therefore, the Equation (12) is satisfied if and only if $\log \sigma_{k}(g)-\lambda=0$ holds. This conclude that $\sigma_{k}$-curvature $\log \sigma_{k}(g)$ is constant as $\lambda$ is constant.

Theorem 3. Any $k$-Yamabe soliton $\left(M, g, v^{T}, \lambda\right)$ on hypersurfaces in $\mathbb{R}^{n+1}$ is contained either in a hypersphere or a hyperplane. 
Proof. Let $\left\{e_{1}, \ldots, e_{n}\right\}$ be an orthonormal frame on $M^{n}$. Assuming that $\varphi$ is any support function on $M^{n}$ and denoting by $\alpha$ the mean curvature, then $\varphi=\tilde{g}(\xi, v)$ for any unit normal vector field $\xi$ and a position vector $v$, and $\mathbf{H}=\alpha \xi$. From Theorem 2, we have

$$
\begin{aligned}
\left(\log \sigma_{k}(g)-\lambda-1\right) g_{i j} & =\tilde{g}\left(h\left(e_{i}, e_{j}\right), v^{\tilde{\xi}}\right)=\tilde{g}\left(g\left(A_{\xi}\left(e_{i}\right), e_{j}\right) \xi, v\right) \\
& =\tilde{g}\left(\kappa_{i} g_{i j} \xi, v\right) \\
& =\kappa_{i} g_{i j} \varphi,
\end{aligned}
$$

such that $h\left(e_{i}, e_{j}\right)=g\left(A_{\xi}\left(e_{i}\right), e_{j}\right) \xi=\kappa_{i} g_{i j} \xi$, where $A_{\xi}\left(e_{i}\right)=\kappa_{i} e_{i}(i=1, \ldots, n)$, and $\kappa_{i}$ is a principle curvature. Therefore, from the above equation, we get:

$$
n\left(\log \sigma_{k}(g)-\lambda-1\right)=\kappa_{i} \varphi .
$$

Tracing the above equation, we finally get

$$
\log \sigma_{k}(g)-\lambda-1=\varphi \alpha .
$$

Combining (20) and (21), we get:

$$
\kappa_{i}=\frac{1}{n} \alpha .
$$

This implies that $M^{n}$ is totally umblical submanifold and hence $h$ satisfies $h\left(W_{1}, W_{2}\right)=$ $\frac{1}{n} \alpha g\left(W_{1}, W_{2}\right) \xi$. Now, let $0=\tilde{\nabla}_{W_{1}} \tilde{g}(\xi, \xi)=2 \tilde{g}\left(\nabla_{W_{1}} \xi, \xi\right)=2 \tilde{g}\left(\nabla_{W_{1}}^{\perp} \xi, \xi\right)$. This shows that the normal connection $\nabla_{W_{1}}^{\perp} \xi$ is flat, i.e., $\nabla_{W_{1}}^{\perp} \xi=0$ and hence $\xi$ is constant. Then, the covariant derivative $\tilde{\nabla}_{W_{1}} h$ is given by

$$
\begin{aligned}
\left(\tilde{\nabla}_{W_{1}} h\right)\left(W_{2}, W_{3}\right) & =\nabla_{W_{1}}^{\perp} h\left(W_{2}, W_{3}\right)-h\left(\nabla_{W_{1}} W_{2}, W_{3}\right)-h\left(\nabla_{W_{1}} W_{3}, W_{2}\right) \\
& =\frac{1}{n} W_{1}(\alpha) g\left(W_{2}, W_{3}\right) \xi
\end{aligned}
$$

for any vector fields $W_{1}, W_{2}, W_{3}$ are tangent to $M^{n}$. From the Codazzi equation and the fact that $R^{\perp}$ is flat, one obtains:

$$
\left(\tilde{\nabla}_{W_{1}} h\right)\left(W_{2}, W_{3}\right)=\left(\tilde{\nabla}_{W_{2}} h\right)\left(W_{1}, W_{3}\right) .
$$

From (23) and (24), we found that $W_{1}(\alpha) W_{2}=W_{2}(\alpha) W_{1}$. This means that $W_{1}$ and $W_{2}$ are linearly independents and hence $\alpha$ is a constant. First, we consider $\alpha=0$, then using the fact that $\tilde{\nabla}_{W_{1}} \xi=-A_{\xi}\left(W_{1}\right)=-\frac{\alpha}{n} W_{1}=0, \xi$ is restricted to $M^{n}$, and $\alpha$ is constant in $\mathbb{R}^{n}$, thus we define

$$
\begin{aligned}
\tilde{\nabla}_{W_{1}} \tilde{g}(v, \xi) & =\tilde{g}\left(\tilde{\nabla}_{W_{1}} v, \xi\right)+\tilde{g}\left(v, \tilde{\nabla}_{W_{1}} \xi\right) \\
& =\tilde{g}\left(W_{1}, \xi\right) \\
& =0 .
\end{aligned}
$$

We conclude that $\tilde{g}(v, \xi)$ is constant in $\mathbb{R}^{n+1}$ such that $v$ and $\xi$ are defined on $M^{n}$. Hence, $M^{n}$ is contained in hyper-plane perpendicular to $\xi$. On the other hand, if $\alpha \neq 0$, then we define

$$
\begin{aligned}
\tilde{\nabla}_{W_{1}}\left(v+n \alpha^{-1} \xi\right) & =\tilde{\nabla}_{W_{1}} v+n \alpha^{-1} \tilde{\nabla}_{W_{1}} \xi \\
& =W_{1}+n \alpha^{-1}\left(-A_{W_{1}}(\xi)\right) \\
& =W_{1}-W_{1} \\
& =0 .
\end{aligned}
$$


Therefore, the vector field $v+n \alpha^{-1} \xi$ is restricted to $M^{n}$ and degenerates to be a constant in $\mathbb{R}^{n+1}$. This shows that $M^{n}$ is contained in the hypersphere. The further part follows from $[18,23]$. This completes the proof of the theorem.

Another interesting result is the following one:

Theorem 4. If a $k$-Yamabe soliton $\left(M^{n}, g, v^{T}, \lambda\right)$ on a submanifold $M^{n}$ of a Riemannian manifold $\widetilde{M}$ is minimal, then $\log \sigma_{k}(g)$ is constant.

Proof. Let $\left\{e_{1}, \ldots, e_{n}\right\}$ be an orthonormal frame on $M^{n}$.

$$
\left(\log \sigma_{k}(g)-\lambda-1\right) g_{i j}=g\left(A_{v \xi}\left(e_{i}\right), e_{j}\right)
$$

From the Equation (20), we have $n\left(\log \sigma_{k}(g)-\lambda-1\right)=\alpha \varphi$ and since we have that $\alpha \varphi=\tilde{g}\left(\mathbf{H}, v^{\xi}\right)$, one obtains:

$$
n\left(\log \sigma_{k}(g)-\lambda-1\right)=\tilde{g}\left(\mathbf{H}, v^{\tilde{\xi}}\right)
$$

As we assumed that $\left(M^{n}, g, v^{T}, \lambda\right)$ is minimal, then $\log \sigma_{k}(g)=\lambda+1$. It means that $\log \sigma_{k}(g)$ is constant as $\lambda$ is constant.

In [24], authors showed that the canonical vector field $v^{T}$ of a submanifold $M$ of the Euclidean $m$-space $\mathbb{R}^{m}$ is a conformal vector field if and only if $M$ is umbilical with respect to the normal component $v^{\tau}$ of the position vector field. Using this concept and Theorem 2, we give the following result.

Corollary 2. If an Euclidean submanifold of $(M, g)$ of $\mathbb{R}^{m}$ is a $k$-Yamabe solitons with canonical vector field $v^{T}$ as its soliton vector field, then $v^{T}$ is a conformal vector field.

Proof. Assuming that the canonical vector field $v^{T}$ is a soliton vector field, then from (12), we have

$$
\tilde{g}\left(h\left(W_{1}, W_{2}\right), v^{\xi}\right)=\left(\log \sigma_{k}(g)-\lambda-1\right) g\left(W_{1}, W_{2}\right)
$$

for the vectors $W_{1}, W_{2}$ which are tangent to $M$. On the other hand, we have

$$
A_{v \xi}\left(W_{1}\right)=\left(\log \sigma_{k}(g)-\lambda-1\right) W_{1}
$$

and $\lambda$ is a constant, then $M$ is umbilical with respect to $v^{\xi}$. Now, applying Theorem 3.1 to [24], we conclude that $v^{T}$ is a conformal vector field.

We obtain following corollary

Corollary 3. The scalar curvature of a compact $k$-Yamabe solitons on a minimal submanifold in $\tilde{M}$ is vanished

Proof. For a minimal submanifold and using Theorem 4 , we have $\log \sigma_{k}(g)=\lambda+1$. Since from (3), we get $\Delta f=n$. This we get $f$ is a constant function by implementing the maximum principle. From [2], we have the following

$$
(n-1) \Delta \log \sigma_{k}(g)+\frac{1}{2} g(\nabla R, \nabla f)+R\left(\log \sigma_{k}(g)-\lambda\right)=0 .
$$

From the above equation we get $R=0$ as $f$ is a constant.

\section{Gradient $k$-Yamabe Soliton}

In this section, we will study about gradient $k$-Yamabe Soliton with the Hodge-de Rham decomposition 
Theorem 5. Let $\left(M^{n}, g, v^{T}, \lambda\right)$ be a $k$-Yamabe soliton on submanifold $M^{n}$ of Riemannian manifold $\widetilde{M}$. Then $\left(M^{n}, g, v^{T}, \lambda\right)$ is a gradient $k$-Yamabe soliton.

Proof. Let us consider that $2 f=\tilde{g}(v, v)$. Thus

$$
g\left(\nabla f, W_{1}\right)=W_{1}(f)=\frac{1}{2} W_{1} g(v, v)=g\left(v, \nabla_{W_{1}} v\right) .
$$

As $v$ is concurrent vector field then we arrive at

$$
g\left(\nabla f, W_{1}\right)=\tilde{g}\left(v, W_{1}\right) .
$$

This means that if $\nabla f=v^{T}$, then the soliton vector field $v^{T}$ is a gradient vector field. Hence, $\left(M^{n}, g, v^{T}, \lambda\right)$ is a gradient $k$-Yamabe soliton. This completes the proof of the theorem.

Corollary 4. If the scalar curvature $R$ of a compact gradient $k$-Yamabe soliton is constant then $k$-curvature $\log \sigma_{k}(g)$ is also constant.

Proof. Taking integration in (27) and using Stokes Theorem, we get

$$
\int g(\nabla R, \nabla f) d V=2 \int R\left(\lambda-\log \sigma_{k}(g)\right) d V .
$$

As we assumed that the scalar curvature $R$ is constant from above equation, we get

$$
R \int\left(\lambda-\log \sigma_{k}(g)\right) d V=0 .
$$

This implies that $\lambda=\log \sigma_{k}(g)$ and hence $\log \sigma_{k}(g)$ is constant.

Theorem 6. Let $(M, g, \nabla f, X, \lambda)$ be a gradient $k$-Yamabe soliton of dimension $n$ which is compact, with potential function $f$. Then upto a constant, $f$ agrees with the Hodge-de Rham potential $\omega$.

Proof. It follows that from (2) for a $k$-Yamabe soliton $(M, g, \nabla f, X, \lambda)$

$$
\left(\log \sigma_{k}(g)-\lambda\right) n=\operatorname{div} X .
$$

Then from Hodge-de-Rham decomposition (10) along with (11), we get

$$
\operatorname{div} X=\Delta \omega .
$$

Therefore, we obtain the following from (28)

$$
\left(\log \sigma_{k}(g)-\lambda\right) n=\Delta \omega
$$

Tracing Equation (3), we derive

$$
\left(\log \sigma_{k}(g)-\lambda\right) n=\Delta f .
$$

Combining (29) and (30), we get the following

$$
\Delta(f-\omega)=0 .
$$

From the above it implies that $f=\omega+C$ for any constant $C$. The proof is completed.

Leyang et al. [2] defined the following example for a $k$-Yamabe soliton. 
Example 1. [2] Assuming the cylinder $\mathbb{S}^{n-1} \times \mathbb{R}$ having the metric

$$
g=d r^{2}+\frac{1}{2}\left(B_{n-1}^{k}-B_{n-1}^{k-1}\right)^{\frac{1}{k}} e^{-\frac{\lambda(t)}{k}} g_{\mathbb{S}^{n-1}}
$$

such that $\lambda$ is a function which depend on $t$. Now consider $k<\frac{n}{2}$ for positive $k$-curvature and boundary curvature $B^{k}$ [25]. For indices $i, j, k(2 \leq i, j, k, \leq n)$ and $r$ denote the direction, we assume that $C=\frac{1}{2}\left(B_{n-1}^{k}-B_{n-1}^{k-1}\right)^{\frac{1}{k}} e^{-\frac{\lambda(t)}{k}} g_{\mathbb{S}^{n-1}}$, then scalar curvature, Ricci curvature and the Schouten tensor of the metric $g$ are defined as follows:

$$
\begin{aligned}
R & =C^{-1}(n-1)(n-1) \\
R i c_{i j} & =(n-2)\left(g_{\mathbb{S}^{n-1}}\right)_{i j}, \quad \operatorname{Ric}_{i r}=R i c_{r r}=0 \\
A_{i j} & =\frac{1}{2}\left(g_{\mathbb{S}^{n-1}}\right)_{i j} A_{i r}=0, \quad A_{r r}=-\frac{1}{2 C} .
\end{aligned}
$$

Taking into account the above relations, the $k$-curvature is obtained as

$$
\sigma_{k}\left(g^{-1} A\right)=B_{n-1}^{k}\left(\frac{1}{2 C}\right)^{k}-B_{n-1}^{k-1}\left(\frac{1}{2 C}\right)^{k}=e^{\lambda(t)} .
$$

It is constant at any fixed time $t$. Therefore, we have

$$
\nabla^{2} f=0=\left(\log \sigma_{k}(g)-\lambda(t)\right) g
$$

for the potential function $f=c r$ where $c$ is any constant. This shows that for any $k$-Yammabe soliton on a complete noncompact manifold, the condition of constant $k$-curvature does not imply that the potential function $f$ is constant.

\section{Conclusions}

In the present paper, we studied $k$-Yamabe soliton which is a natural extension of the Yamabe flow where the evolving metric satisfies the partial differential Equation (1). In the differential geometry, for the $k$-Yamabe soliton, an important question is to find conditions under which this soliton become a trivial $k$-Yamabe solitons and also a gradient $k$-Yamabe soliton. On the other hand, the geometric flows represents a topic of active research interest in both mathematics and physics. One of the well-known geometric flows in mathematics is the heat flow [26]. Stable solutions of Yamabe flow are said to be Yamabe solitons. The Yamabe flow is an intrinsic geometric flow, a process which deforms the metric of a Riemannian manifold and it was introduced by Richard S. Hamilton. A Yamabe flow is defined for noncompact manifolds and is the negative $L^{2}$-gradient flow of the (normalized) total scalar curvature, restricted to a given conformal class. If this flow converges then, this can be regarded as a deformation of the Riemannian metric to a conformal metric of constant scalar curvature. The main importance of the Hamilton conjecture is that for every initial metric, the flow converges to a conformal metric of constant scalar curvature and later it becomes a central tool in applications to various areas of sciences and economics. Our results are important in this respect.

Author Contributions: Writing and original draft, A.A.; funding acquisition, editing and draft, F.M.; review and editing, N.A.; methodology, project administration, A.A.; formal analysis, resources, N.A. Improving the quality of the paper, review and editing: P.L.-I. All authors have read and agreed to the published version of the manuscript.

Funding: This research was funded by the Deanship of Scientific Research at Princess Nourah bint Abdulrahman University through the Fast-track Research Funding Program.

Institutional Review Board Statement: Not applicable.

Informed Consent Statement: Not applicable. 
Conflicts of Interest: The authors declare no conflict of interest.

\section{References}

1. Besse, A.L. Einstein manifolds. In Classics in Mathematics; Springer: Berlin, Germany, 2008.

2. Leyang, B. The $k$-Yamabe solitons and the quotient Yamabe solitons. Nonlinear Anal. 2018, 166, $181-195$.

3. Tokura, W.; Batista, E. Triviality results for compact $k$-Yamabe solitons. arXiv 2006, arXiv:2006.00501.

4. Chen, B.Y.; Deshmukh, S. Ricci solitons and concurrent vector fields. Balkan J. Geom. Appl. 2015, 20, 14-25.

5. Chen, B.Y.; Deshmukh, S. Classification of Ricci solitons on Euclidean hypersurfaces. Internat. J. Math. 2014, $25,1450104$. [CrossRef]

6. Chen, B.Y.; Deshmukh, S. Yamabe and quasi-Yamabe solitons on Euclidean submanifolds. Mediterr. J. Math. 2018, 15, 194. [CrossRef]

7. Al-Dayel, I.; Deshmukh, S.; Belova, O. A remarkable property of concircular vector fields on a Riemannian manifold. Mathematics 2020, 8, 469. [CrossRef]

8. Alkhaldi, A.H.; Ali, A. Classification of warped product submanifolds in Kenmotsu space forms admitting gradient Ricci solitons. Mathematics 2019, 7, 112. [CrossRef]

9. Shaikh, A.; Mandal, P. Some characterizations of gradient Yamabe solitons. arXiv 2003, arXiv:2003.12445v1.

10. Barbosa, E.; Ribeiro, E. On conformal solutions of the Yamabe flow. Arch.Math. 2013, 101, 79-89. [CrossRef]

11. Nasser, B.T.; Chen, B.Y.; Deshmukh, S. Conformal vector fields and Yamabe solitons. Int. J. Geom. Methods Mod. Phys. 2019, 16, 1950053.

12. Bejan, C.; Crasmareanu, M. Ricci solitons in manifolds with quasi-constant curvature. Publ. Math. Debrecen. 2011, 78, 235-243. [CrossRef]

13. Chen, B.Y. Topics in differential geometry associated with position vector fields on Euclidean submanifolds. Arab J. Math. Sci. 2017, 23, 1-17. [CrossRef]

14. Deshmukh, S.; Alsodais, H. A note on Ricci solitons. Symmetry 2020, 12, 289. [CrossRef]

15. Deshmukh, S.; Peska, P.; Turki, N.B. Geodesic vector fields on a Riemannian manifold. Mathematics 2020, 8, 137.

16. Faraji, H.; Azami, S.; Fasihi-Ramandi, G. h-Almost Ricci solitons with concurrent potential fields. AIMS Mathematics 2020, 5, 4220. [CrossRef]

17. Benedito, N.L.; Hudson, P.O. Generalized quasi Yamabe gradient solitons. Diff. Geom. Appl. 2016, 49, $167-175$.

18. Seko, T. Classification of almost Yamabe solitons in Euclidean spaces. J. Geom. Phys. 2019, 136, 97-103. [CrossRef]

19. Chen, B.Y. Geometry of Submanifolds; Marcer Dekker: New York, NY, USA, 1973; ISBN 978-3-0348-0983-2.

20. Samelson, H. Orientability of hypersurfaces in $\mathbb{R}^{n}$. Proc. Am. Math. Soc. 1969, 22, 301-302.

21. Warner, F. Foundations of Differentiable Manifolds and Lie Groups; Springer-Verlag: New York, NY, USA, 1983; ISBN 978-0-387-90894-6.

22. Chen, B.Y. Pseudo-Riemannian Geometry, $\delta$-Invariants and Applications; World Scientific: Hackensack, NJ, USA, 2011; ISBN 978-981-4462-48-8.

23. Fujii, S.; Maeta, S. Classification of generalized Yamabe solitons in Euclidean spaces. arXiv 2009, arXiv:2009.13255.

24. Chen, B.Y.; Deshmukh, S. Euclidean submanifolds with conformal canonical vector field. Bull. Korean Math. Soc. 2018, 55, 1823-1834.

25. Chen, S. Conformal deformation on manifolds with boundary. Geom. Funct. Anal. 2009, 19, 1029-1064. [CrossRef]

26. Brendle, S. Convergence of the Yamabe flow for arbitrary initial energy. J. Diff. Geom. 2005, 69, 217-278. [CrossRef] 